\title{
IDENTIFICATION OF X AND Y SPERMATOZOA IN THE NORTHERN VOLE, MICROTUS OECONOMUS
}

\author{
A. D. TATES,* P. L. PEARSON AND J. P. M. GERAEDTS \\ Department of Radiation Genetics and Chemical Mutagenesis, and \\ *Department of Human Genetics, Medical Faculty, University of Leiden, \\ The Netherlands
}

(Received 9th August 1974)

The introduction of a quinacrine staining technique for identifying human chromosomes (Zech, 1969) also permitted recognition of the human Y chromosome in non-dividing cells (Pearson, Bobrow \& Vosa, 1970), including spermatozoa (Pearson \& Bobrow, 1970; Barlow \& Vosa, 1970). The ability to distinguish between human $\mathrm{X}$ - and Y-bearing spermatozoa has been used in estimating the difference in the DNA content of these spermatozoa (Sumner, Robinson \& Evans, 1971; Pearson, Geraedts \& Pawlowitzki, 1973), the primary non-disjunctional frequency of the Y chromosome (Sumner, Robinson \& Evans, 1971; Pawlowitzki \& Pearson, 1972) and, more recently, for judging the success of a separation technique for $\mathrm{X}$ and $\mathrm{Y}$ spermatozoa (Ericsson, Langevin \& Nishino, 1973). Chromosomes 1 and 9 have also been recognized in human spermatozoa (Pearson, 1972; Bobrow, Madan \& Pearson, 1972; Geraedts \& Pearson, 1973) by using variations in C-banding techniques which specifically stain the centric heterochromatin regions of these two chromosomes. There appear to be certain similarities in the staining properties of the chromosomes which have so far been detected in spermatozoa. The following criteria are accepted for recognition of chromosomes in spermatozoa: (a) there is a region of centric heterochromatin localized on one particular chromosome which can be stained and recognized uniquely in mitotic chromosome preparations; (b) the heterochromatic region remains visible in interphase nuclei as a differentially stained body; and (c) the latter is also visible through all the meiotic series into mature spermatozoa. As yet, direct cytological observations have only permitted an identification of X-and Y-bearing gametes and an estimate of the frequencies with which non-disjunctional events occur in human spermatozoa. Recently, Benirschke (1973) has emphasized the necessity for developing a sperm-staining system in other animals comparable to that in the human so that the effect of environmental agents, such as radiation, drugs and synthetic food additives, on non-disjunctional frequencies can be directly assessed.

As part of an exploratory programme for mammalian species with karyotypes suitable for permitting chromosome identification in their spermatozoa, we first confined our attention to those species known to have either a single, or at most two, large regions of constitutive heterochromatin in their karyotype. In principle, such regions should be demonstrable in spermatozoa by using a normal C-banding technique and this should not necessitate developing a 
specialized technique to distinguish a heterochromatic region on one particular chromosome from the regions on other chromosomes. Voles of the species Microtus oeconomus appeared to have such a large single region, specifically a completely heterochromatic Y chromosome (U. Wolf, personal communication). A breeding colony was established in Leiden from two pairs of animals that had been captured in Northern Sweden. After several months, two males of proven fertility were taken from the colony and used in the present study. Here, we present results showing that the X-and Y-bearing spermatids and spermatozoa, and also non-disjunctional events involving these chromosomes, can be recognized in $M$. oeconomus.

Both animals were injected intraperitoneally with $160 \mu \mathrm{g}$ Colcemid. Following cervical dislocation $2 \mathrm{hr}$ later, mitotic chromosome preparations were prepared from the bone marrow and meiotic chromosome preparations from the testes. The air-dried preparations were left for 4 days and were then treated as for a standard C-banding technique by first heating the slides in boiling distilled water for $12 \mathrm{~min}$, then standing them in two changes of standard saline citrate at $65^{\circ} \mathrm{C}$ for $4 \mathrm{hr}$ and finally rinsing in distilled water and staining with a $0.5 \%$ Leishman solution at $\mathrm{pH} 6.8$ for $12 \mathrm{~min}$.

The bone marrow metaphases showed a single completely heterochromatic Y chromosome: small blocks of heterochromatin were also present on most of the other chromosomes. This pattern was confirmed in the meiotic preparations in which, at diakinesis, an overall darkly staining $\mathrm{Y}$ chromosome was attached to the long arm of the $\mathrm{X}$ chromosome, and the much smaller heterochromatic regions of the $\mathrm{X}$ chromosome and the autosomes were also visible in the bivalents (Pl. 1, Fig. 1). In early and mid-term spermatids, two types of cells were apparent, one with a distinct and darkly staining block which we regarded as the interphase $\mathrm{Y}$ chromosome and the other with a larger, more diffuse and lighter stained body which we interpreted as either the $\mathrm{X}$ chromosome or a structure associated with the $\mathrm{X}$ chromosome (Pl. 1, Figs 2 and 3). The latter observation was somewhat surprising because the size of the X-body was far too large to be accounted for by the amount of X-borne centric heterochromatin seen in either bone marrow mitoses or at diakinesis. It is possible that the body represents some form of premature condensation product of the $\mathrm{X}$ chromosome. In testicular spermatozoa, we can recognize the $\mathrm{Y}$ chromosome

\section{EXPLANATION OF PLATE 1}

FIG. 1. Diakinesis in a meiotic preparation of $M$. oeconomus testis, illustrating the heterochromatic $Y$ chromosome (small arrow) and the centric heterochromatin of the $X$ chromosome (large arrow). $\times 1400$.

FIG. 2. Early spermatids with dense $Y$ bodies (small arrows) and larger, more diffuse $X$ bodies (large arrows). $\times 1400$.

Fig. 3. Intermediate spermatids with dense $\mathrm{Y}$ bodies (small arrows) and less dense $\mathrm{X}$ bodies (large arrows) $\times 1400$.

Fig. 4. Mature spermatozoa showing $\mathrm{Y}$ bodies (small arrows) and other spermatozoa without bodies (large arrows). $\times 1400$.

FIg. 5. Young non-disjunctional spermatid with two $\mathrm{Y}$ bodies (double small arrow). $\times 1400$.

Fic. 6. Diploid young spermatid with an $X$ and $Y$ body (arrowed). $\times 1400$. 


\section{PLATE}

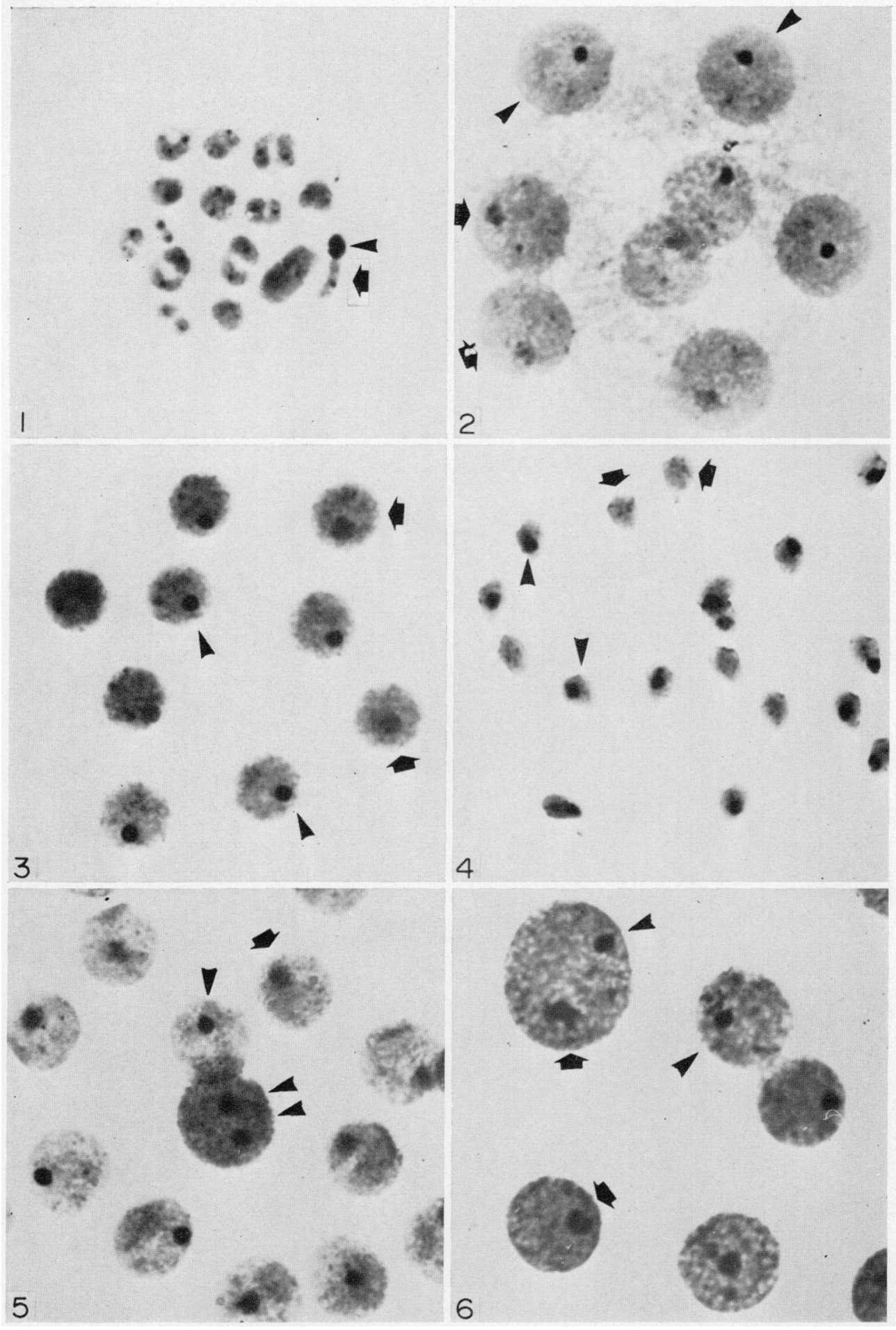

(Facing p. 196) 
but usually can no longer identify the X chromosome (Pl. 1, Fig. 4). The Ybodies in the spermatozoa, although similar in size to those found in other cell types and tissues, appear to occupy a large proportion of the nuclear volume. This is similar to the situation found in human spermatozoa for chromosomes 1 and 9 for which C-banding techniques are also used. Within the limitations of locating variations in the position of a relatively large mass within a confined area, there does not appear to be a preferred location for the $\mathrm{Y}$ chromosome within the sperm nucleus. In some instances, the Y-body was a bifid structure and resembled that found in some human spermatozoa.

Table 1. The frequencies of normal and non-disjunctional gametes in two male $M$. oeconomus

\begin{tabular}{c|c|c}
\hline Cell type & Animal 1 & Animal 2 \\
\hline Y & 388 & 401 \\
X & 350 & 382 \\
YY & 2 & 2 \\
YX & 1 & 1 \\
XX & 1 & - \\
\hline
\end{tabular}

Frequencies of $\mathrm{X}$ and $\mathrm{Y}$ gametes have been calculated from spermatids and are shown in Table 1. The frequencies of non-disjunctional gametes (Pl. 1, Fig. 5) are also shown in Table 1 and it is clear that the non-disjunctional frequency for $\mathrm{Y}-\mathrm{Y}$ gametes is much smaller than that calculated for man. In $M$. oeconomus, we have the added advantage that we can also recognize other combinations of sex chromosome non-disjunction. A small proportion of the non-disjunctional gametes found in $M$. oeconomus are much larger and appear to be polyploid (Pl. 1, Fig. 6). This has been confirmed in several instances by scanning microdensitometry.

The convenient term 'non-disjunctional' has been used in this contribution to refer to all forms of aneuploid spermatozoa irrespective of their meiotic origin. Formally, however, non-disjunctional gametes can only arise in instances when there is inadequate separation following synapsis and would not include examples of asynapsis.

We are continuing our work on $M$. occonomus by collecting more information on spontaneous and induced non-disjunctional frequencies to assess the value of this species as a screening tool in mutagenicity testing. Moreover, the development of an X-and Y-bearing sperm identification technique in a species other than man now increases the scope for those laboratories interested in developing methods of separating such spermatozoa.

The work reported in this paper received support from the Institute for Radiopathology and Radiation Protection. The authors want to thank Professor F. H. Sobels for his interest in the project and Mr F. Leupe for his help in setting up and maintaining the breeding colony of $M$. oeconomus. The two pairs of $M$. oeconomus from which the Leiden breeding colony was established were kindly provided by $\mathrm{Dr} \mathrm{K}$. Fredga. 


\section{REFERENGES}

Barlow, P. \& Vosa, C. G. (1970) The Y chromosome in human spermatozoa. Nature, Lond. 226, 961-962.

Bobrow, M., Madan, K. \& Pearson, P. L. (1972) Staining of some specific regions of human chromosomes, particularly the secondary constriction of No. 9. Nature, New Biol. 238, 122-124.

Benirschke, K. (1973) Conclusions. In Les Accidents Chromosomiques de la Reproduction, pp. 405-413. Eds. A. Boué and C. Thibault. INSERM, Paris.

Ericsson, R. J., Langevin, C. N. \& Nishino, M. (1973) Isolation of fractions rich in human Y sperm. Nature, Lond. 246, 421-424.

Geraedts, J. P. M. \& Pearson, P. L. (1973) Specific staining of the human No. 1 chromosome in spermatozoa. Humangenetik, 20, 171-173.

Pawlowitzki, I. H. \& Pearson, P. L. (1972) Chromosomal aneuploidy in human spermatozoa. Humangenetik, 16, 119-122.

Pearson, P. L. (1972) The use of new staining techniques for human chromosome identification. J. med. Genet. 9, 264-275.

Pearson, P. L. \& Bobrow, M. (1970) Fluorescent staining of the Y chromosome in meiotic stages of the human male. 7 . Reprod. Fert. 22, 177-179.

Pearson, P. L.., Bobrow, M. \& Vosa, C. G. (1970) Technique for identifying Y chromosomes in human interphase nuclei. Nature, Lond. 226, 78-80.

Pearson, P. L., Geraedts, J. P. M. \& Pawlowitzki, I. H. (1973) Chromosomal studies on human male gametes. In Les Accidents Chromosomiques de la Reproduction, pp. 219-229. Eds. A. Boué and C. Thibault. INSERM, Paris.

Sumner, A. T., Robinson, J. A. \& Evans, H. J. (1971) Distinguishing between X, Y and YY-bearing human spermatozoa by fluorescence and DNA content. Nature, New Biol. 229, 231-233.

$\mathrm{ZECH}, \mathrm{L}$. (1969) Investigation of metaphase chromosomes with DNA-binding fluorochromes. Expl Cell Res. 58, 463. 